\title{
A Crossing Lemma for Annular Regions and Invariant Sets with an Application to Planar Dynamical Systems
}

\author{
Anna Pascoletti and Fabio Zanolin \\ Dipartimento di Matematica e Informatica, Università di Udine, Via delle Scienze 206, 33100 Udine, Italy \\ Correspondence should be addressed to Fabio Zanolin; fabio.zanolin@uniud.it
}

Received 31 January 2013; Accepted 17 June 2013

Academic Editor: Alfredo Peris

Copyright ( 2013 A. Pascoletti and F. Zanolin. This is an open access article distributed under the Creative Commons Attribution License, which permits unrestricted use, distribution, and reproduction in any medium, provided the original work is properly cited.

\begin{abstract}
We present a topological result, named crossing lemma, dealing with the existence of a continuum which crosses a topological space between a pair of "opposite" sides. This topological lemma allows us to obtain some fixed point results. In the works of Pascoletti et al., 2008, and Pascoletti and Zanolin, 2010, we have widely exposed the crossing lemma for planar regions homeomorphic to a square, and we have also presented some possible applications to the theory of topological horseshoes and to the study of chaotic-like dynamics for planar maps. In this work, we move from the framework of the generalized rectangles to two other settings (annular regions and invariant sets), trying to obtain similar results. An application to a model of fluid mixing is given.
\end{abstract}

\section{Introduction}

In 1883-1884, Poincaré $[1,2]$ introduced a generalization of the Bolzano theorem to continuous vector fields defined on a hypercube of $\mathbb{R}^{N}$. Such result, usually known as the PoincaréMiranda theorem [3-5], in dimension two, reads as follows.

Theorem 1. Let $\mathscr{R}:=\left[a_{1}, b_{1}\right] \times\left[a_{2}, b_{2}\right]$ be a planar rectangle, and let $f:=\left(f_{1}, f_{2}\right): \mathscr{R} \rightarrow \mathbb{R}^{2}$ be a continuous vector field such that $f_{1}$ changes its sign from the left to the right side of $\mathscr{R}$ and $f_{2}$ changes its sign from the lower to the upper side of $\mathscr{R}$. Then, there exists at least one point $w \in \mathscr{R}$ such that $f(w)=0$.

A heuristic proof of this result, "pour faire comprendre comment on peut démontrer ce théorème," quoting Poincarés words [2], can be described as follows. (The more formal proof in [2] makes use of Kronecker integral, a forerunner of topological degree theory (see $[5,6])$. We also recommend the recent article [7] for a modern perspective about this topic.) The "curve" $f_{2}\left(x_{1}, x_{2}\right)=0$ starts at some point of the left side $x_{1}=a_{1}$, and it ends at some point of the right side $x_{1}=b_{1}$. Similarly, the "curve" $f_{1}\left(x_{1}, x_{2}\right)=0$ starts at some point of the lower side $x_{2}=a_{2}$, and it ends at some point of the upper side $x_{2}=b_{2}$. Hence, these "curves" must intersect at some point of the rectangle. Clearly, in modern language, using in this context the term "curve" may be a little misleading, although it well explains the geometry of the problem. Yet the argument of the proof is safe if we note that the set $f_{2}\left(x_{1}, x_{2}\right)=0$ contains a continuum joining the left to the right side and, similarly, the set $f_{1}\left(x_{1}, x_{2}\right)=0$ contains a continuum joining the lower to the upper side. The existence of at least one intersection point between these continua follows from a result of plane topology (see Lemma 7).

In order to prove the existence of such continua, one can observe that the set $f_{2}\left(x_{1}, x_{2}\right)=0$ crosses any path from the lower to the upper side of the rectangle (and the same happens for the set $f_{1}\left(x_{1}, x_{2}\right)=0$ with respect to the paths connecting the left to the right side of the rectangle). In [8] we called such kind of results as crossing lemmas and proposed new applications to the study of the dynamics of some planar maps. In an abstract setting we can describe these results as follows. We start from a closed set $\mathcal{S}$ which cuts all the paths connecting two given sets, and we look for a closed connected subset $\mathscr{C}$ of $\mathcal{S}$ with the same cutting property. In many applications, it turns out that the set $\mathscr{C}$ crosses the original domain "from one side to the other." A typical example in this direction is presented in Lemma 10 in the framework of topological rectangles, which are topological spaces which are homeomorphic to a planar rectangle. 
Important theorems, where some forms of these crossing properties are considered, appear in dimension theory with the results of Hurewicz and Wallman [9], in topological games $[10,11]$, and in proofs of the existence of solutions to nonlinear differential equations [12].

In some applications, typically the set $\mathcal{S}$ is a set of solutions of a nonlinear equation depending on a parameter (or equivalently a set of fixed points for a family of parameterdependent operators). From this point of view, results in this direction, in spite of the fact that they may look quite elementary, present a great usefulness in different areas of nonlinear analysis, especially in connection with bifurcation theory (see [12-15]).

When the operators whose fixed points correspond to the element of $\mathcal{S}$ have some special symmetries, it is likely that some of these symmetries are inherited by the set $\delta$ itself. In this case, it would be desirable to prove that also the continuum $\mathscr{C} \subset \mathcal{S}$ inherits the symmetries of $\mathcal{S}$.

In the present paper we move from the framework of generalized rectangles already discussed in previous articles $[8,16]$ to two other settings, namely, the study of annular regions and the case of invariants sets. More precisely, for the former case, we just collect in Section 2 some classical separation results already applied in $[8,16]$ to prove the existence of fixed points, periodic points, and chaotic dynamics for planar dynamical systems. In Section 3 and Section 4, respectively, we discuss the variants to annular regions and invariant sets, and, as a possible application of our results, we reconsider in Section 5 an example of planar map arising in fluid mixing previously studied by Kennedy and Yorke in [17].

We conclude this introduction with some preliminary results which will be used in what follows. Throughout the paper, by a path in a topological space $X$, we mean a continuous map $\gamma: I \rightarrow X$, with $I:=[0,1]$. We also set $\bar{\gamma}:=\gamma(I)$. Any homeomorphic image of $I$ will be referred to as an arc. The sets $B(0, r)$ and $B[0, r]$ are, respectively, the open and the closed balls of center of origin and radius $r$ in $\mathbb{R}^{N}$. In the plane $\mathbb{R}^{2}$ (usually identified with $\mathbb{C}$ ) with the Euclidean norm, we denote by $E^{2}:=B[0,1]$ the closed unit disc and by $S^{1}=\partial E^{2}$ its boundary.

Slightly modifying an analogous definition of Berarducci et al. [18, Definition 2.1], we give the following.

Definition 2. Let $X$ be a topological space, and let $A, B \subset X$ be two nonempty disjoint sets. Let also $S \subset X$. One says that $S$ cuts the paths between $A$ and $B$ if $S \cap \bar{\gamma} \neq \emptyset$, for every path $\gamma: I \rightarrow X$ such that $\gamma(0) \in A$ and $\gamma(1) \in B$.

In order to simplify the statements of the next results, we write $S: A+B$ to express the fact that $S$ cuts the paths between $A$ and $B$. If $A=\{a\}$ is a singleton, we write $S: a+B$ instead of $S:\{a\}+B$. Similar notation is used if $B$ is a singleton. In order to have Definition 2 meaningful, we implicitly assume that there exists at least a path $\gamma$ in $X$ connecting $A$ with $B$ (otherwise, we could take $S=\emptyset$ or $S$ any subset of $X$ ). Clearly, if a set $S$ satisfies the cutting property of Definition 2, then also its closure $\operatorname{cl} S$ cuts the paths between $A$ and $B$. Therefore, in the sequel and without loss of generality, we usually assume $S$ is closed. Such an assumption is also well suited for proving the existence of minimal (closed) sets satisfying Definition 2. Indeed, we have the following.

Lemma 3. Let $X$ be a topological spac,e and let $A, B \subset X$ be two nonempty disjoint sets which are connected by at least one path in $X$. Let $S \subset X$ be a (nonempty) closed set which cuts the paths between $A$ and $B$. Then there exists a nonempty, closed set $C \subset S$ which is minimal with respect to the property of cutting the paths between $A$ and $B$.

Proof. The proof is a standard application of Zorn's lemma. Let $\mathscr{F}$ be the set of all the nonempty closed subsets $F$ of $S$ such that $F: A+B$, with the elements of $\mathscr{F}$ ordered by inclusion. Clearly, $\mathscr{F}$ is nonempty for at least $S \in \mathscr{F}$. Let $\left(F_{\alpha}\right)_{\alpha \in J}$ be a totally ordered subset of $\mathscr{F}$. We claim that $F^{*}:=\bigcap_{\alpha \in J} F_{\alpha} \in$ $\mathscr{F}$. Indeed, let $\gamma: I \rightarrow X$ be a path such that $\gamma(0) \in A$ and $\gamma(1) \in B$. The family of compact sets $\left(\bar{\gamma} \cap F_{\alpha}\right)_{\alpha \in J}$ has the finite intersection property, and, therefore, $\bar{\gamma} \cap \bigcap_{\alpha \in J} F_{\alpha}=\bar{\gamma} \cap F^{*} \neq \emptyset$. This proves our claim, and the conclusion follows from Zorn's lemma.

In [19], Dolcher studied a similar minimality problem, dealing with closed sets separating two points. The definition of separation is the standard one; that is, a set $S \subset X$ separates two points (or, in general, two nonempty sets) if the two points belong to different components of the complement $X \backslash S$. In this respect, we reconsider the following example from [19]. Let $X \subset \mathbb{R}^{2}$, with the topology of the plane, be defined by

$$
X:=\left\{(x, y): x \geq 0, y=\frac{x}{n}, n \in \mathbb{N}_{0}\right\} \cup\left(\mathbb{R}^{+} \times\{0\}\right),
$$

with $\mathbb{R}^{+}:=[0,+\infty)$ and $\mathbb{N}_{0}:=\mathbb{N} \backslash\{0\}$.

Let $A:=\{(0,0)\}, B:=\{(2,0)\}$, and $S:=\{(x, y) \in X$ : $x=1\}$. Clearly, $S: A+B$, and also $S$ separates $A$ and $B$ in $X$. As shown in [19], there is no subset of $S$ which is minimal for the property of separating $A$ and $B$ in $X$. On the other hand, $C:=\{(1,0)\}$ is the minimal subset of $S$ which cuts the paths between $A$ and $B$.

In general, a set $S$ satisfying $S: A+B$, even if minimal with respect to such property, need not be connected. For an elementary example, one can take $X:=S^{1}$ (with the topology of the plane), $A:=\{(-1,0)\}, B:=\{(1,0)\}$, and $S:=\{(0,-1),(0,1)\}$. In this case, $S$ is a closed set, minimal with respect to the property of cutting the paths between $A$ and $B$, which is not connected. The connectivity of $S$ (or of a minimal subset of it) is, however, an important property for the proof of the existence of fixed points or of zeros for maps in Euclidean spaces. Such connectivity properties have been employed recently in [20-22] in connection with the theory of topological horseshoes. In order to recall some main results from the above quoted papers and to propose some further developments, we introduce, in the next section, some main definitions which play a crucial role in our approach. 


\section{Generalized Rectangles}

Definition 4. A topological space $X$ is called a generalized rectangle if it is homeomorphic to the unit square $Q:=I^{2}$.

For a generalized rectangle $X$ and a homeomorphism $\eta: Q \rightarrow \eta(Q)=X$, the set $\eta(\partial Q)$ is independent of the choice of the homeomorphism $\eta$. We call the set $\eta(\partial Q)$ the contour of $X$ and denote it by $\vartheta X$. Clearly, for a generalized rectangle $X$ embedded in $\mathbb{R}^{2}$, the contour of $X$ coincides with the boundary of $X$.

Definition 5. An oriented rectangle is a pair $\widetilde{X}:=\left(X, X^{-}\right)$ such that $X$ is a generalized rectangle and $X^{-} \subset \vartheta X$ is the union of two disjoint $\operatorname{arcs} X_{l}^{-}$and $X_{r}^{-}$. One says that $X_{l}^{-}$ and $X_{r}^{-}$are, respectively, the left- and the right-hand sides of the (generalized) rectangle $X$. Sometimes one will also write $X:=|\widetilde{X}|$.

Remark 6. Given an oriented rectangle, $\widetilde{X}=\left(X, X^{-}\right)$, we can choose a homeomorphism $\eta: Q \rightarrow \eta(Q)=X$ in such a manner that

$$
X_{l}^{-}=\eta(\{0\} \times I), \quad X_{r}^{-}=\eta(\{1\} \times I)
$$

(this can be proved using the Schoenflies theorem [23]). Then we can also introduce the sets

$$
X_{b}^{+}=\eta(I \times\{0\}), \quad X_{t}^{+}=\eta(I \times\{1\}),
$$

which are called the bottom and the top sides of $\widetilde{X}$ and define

$$
X^{+}:=X_{b}^{+} \cup X_{t}^{+} .
$$

Observe that, for any homeomorphism $\eta_{1}: Q \rightarrow \eta_{1}(Q)=X$ such that $\eta_{1}(\{0,1\} \times I)=X^{-}$, it follows that $\eta_{1}(I \times\{0,1\})=$ $X^{+}$. Thus, the choice of $X^{-}$determines also the choice of $X^{+}$(which is also the closure of $9 X \backslash X^{-}$). Given a pair $\left(X^{-}, X^{+}\right)$, the order in which we choose to call as "right" and "left" to the two components of $X^{-}$(as well as to label the two components of $X^{+}$) is completely irrelevant for what follows. As a consequence of such remark, we have that, to each oriented rectangle $\widetilde{X}$, a "dual" oriented rectangle $\widetilde{X}^{\prime}$ is associated with $|\widetilde{X}|=\left|\widetilde{X}^{\prime}\right|=X$ and with $X^{+}$as the $[\cdot]^{-}$-set for $\widetilde{X}^{\prime}$.

2.1. Crossing Properties for Generalized Rectangles. The next (classical) result guarantees the fact that continua connecting opposite sides of an oriented rectangle must cross each other. It can be proved as a consequence of the Jordan curve theorem, and the proof is omitted (see [16] for the details and also [24] for an application to ordinary differential equations).

Lemma 7. Let $\widetilde{X}=\left(X, X^{-}\right)$be an oriented rectangle, and let $C_{1}, C_{2} \subset X$ be closed connected sets such that

$$
C_{1} \cap X_{l}^{-} \neq \emptyset \neq C_{1} \cap X_{r}^{-}, \quad C_{2} \cap X_{b}^{+} \neq \emptyset \neq C_{2} \cap X_{t}^{+} .
$$

Then $C_{1} \cap C_{2} \neq \emptyset$.
The connectedness of $C_{1}$ and $C_{2}$ is not enough to guarantee the existence of a nonempty intersection (see [25, Example 10.4]).

We present now some results about sets separating the opposite faces of an oriented rectangle. We have shown their role in the proof of the existence of fixed points and periodic points for continuous maps in $[8,16,26]$. Some of these results can be extended to higher dimension using the topological degree, the fixed point index, or other index theories (see [22] and the references therein). Since the applications in the present paper will be all related to planar maps, we prefer to confine ourselves to the use of a more direct tool: Alexander's lemma. Such result, named after Alexander [27, 28], as shown both in Newman's book [29] and in Sanderson's article [30], is quite useful in proving a broad range of theorems of plane topology. Quoting Smith [31] "this lemma, the proof of which requires but a few lines, is shown $[\cdots]$ to be one of the sharpest tools in the theory of separation, if skilfully handled." Results based on applications of Alexander's lemma or to other related theorems in [29] have been fruitfully applied to differential equations by Hastings [32-34], McLeod and Serrin [35], Turner [36], and others. For more recent applications see $[15,37,38]$.

The following version of Alexander's lemma will be used in our next results. The proof requires only an elementary modification of the standard one [39] and, therefore, is omitted.

Lemma 8. Let $\widetilde{X}=\left(X, X^{-}\right)$be an oriented rectangle, and let $K_{1}, K_{2}$ be closed disjoint subsets of $X$. Assume that there exist two paths $\gamma_{1}, \gamma_{2}: I \rightarrow X$, with $\gamma_{1}(0), \gamma_{2}(0) \in X_{l}^{-}$and $\gamma_{1}(1), \gamma_{2}(1) \in X_{r}^{-}$such that

$$
\bar{\gamma}_{1} \cap K_{1}=\emptyset, \quad \bar{\gamma}_{2} \cap K_{2}=\emptyset .
$$

Then, there exists a path $\gamma: I \rightarrow X$, with $\gamma(0) \in X_{l}^{-}$and $\gamma(1) \in X_{r}^{-}$such that $\bar{\gamma} \cap\left(K_{1} \cup K_{2}\right)=\emptyset$.

Remark 9. The original version of Alexander's lemma is a variant of Lemma 8 in which we assume that all the paths have the same initial point $p \in X_{l}^{-}$and the same end point $q \in X_{r}^{-}$.

The next result is perhaps one of the most classical and useful consequences of the previous lemma (see [29]) and is usually expressed by the fact that "if a closed set separates the plane, then some component of this set separates the plane" [34, page 131].

Lemma 10. Let $\widetilde{X}=\left(X, X^{-}\right)$be an oriented rectangle, and let $S \subset X$ be a closed set such that $S: X_{l}^{-}+X_{r}^{-}$. Then, there exists a compact, connected set $C \subset S$ such that $C: X_{l}^{-}+X_{r}^{-}$.

Proof. By Lemma 3 there exists a closed set $C \subset S$ such that $C: X_{l}^{-}+X_{r}^{-}$, with $C$ minimal with respect to the cutting property. Suppose, by contradiction, that $C$ is not connected, and let $C_{1}, C_{2} \subset C$ be two closed nonempty disjoint sets with $C_{1} \cup C_{2}=C$. Since $C$ is minimal and $C_{1}, C_{2}$ are proper subsets of $C$, there exist two paths $\gamma_{1}, \gamma_{2}$ in $X$ which connects $X_{l}^{-}$to $X_{r}^{-}$, such that $\gamma_{i}$ avoids $C_{i}$ (for $i=1,2$ ). Then, by Lemma 8 , there exists a path $\gamma: I \rightarrow X$ with $\gamma(0) \in X_{l}^{-}$and $\gamma(1) \in X_{r}^{-}$ 
with $\bar{\gamma} \cap C=\emptyset$, contradicting the assumption that $C: X_{l}^{-}+$ $X_{r}^{-}$.

The cutting property for $C$ obtained in Lemma 10 can be equivalently expressed as follows.

Lemma 11. Let $\widetilde{X}=\left(X, X^{-}\right)$be an oriented rectangle, and let $C \subset X$ be a closed connected set. Then, $C: X_{l}^{-}+X_{r}^{-}$if and only if $C \cap X_{b}^{+} \neq \emptyset \neq C \cap X_{t}^{+}$.

Proof. If $C: X_{l}^{-}+X_{r}^{-}$, then, necessarily, $C$ must cut the upper and the lower sides of $\widetilde{X}$ which are the images of particular paths connecting $X_{l}^{-}$to $X_{r}^{-}$. On the other hand, if $\gamma: I \rightarrow X$ is any path with $\gamma(0) \in X_{l}^{-}$and $\gamma(1) \in X_{r}^{-}$, then $\bar{\gamma}$ and $C$ are two continua connecting the opposite sides of the oriented rectangle and, therefore, $\bar{\gamma} \cap C \neq \emptyset$ by Lemma 7 . This proves that $C: X_{l}^{-}+X_{r}^{-}$.

Lemma 11, as well as Lemma 7, can be seen as a continuous version of the so-called Hex theorem asserting that Hex cannot end in a tie [11].

The combination of Lemma 10 and Lemma 11 gives the so-called Crossing Lemma asserting the fact that if a closed set intersects all the paths from the left to the right side in an oriented rectangle, then it contains a continuum connecting the two other sides. Note also that such a continuum can be taken as irreducible between $X_{b}^{+}$and $X_{t}^{+}$(by using some classical results from $[13,40])$. See $[16]$ for a recent survey on this subject and its connections with various different results of plane topology, as well as for a different proof based on Whyburn's lemma.

\section{Annular Regions}

In this section, we are moving our attention from generalized rectangles to planar annuli, trying to develop analogous results of Section 2 in this new setting. First of all, we present some basic definitions.

A closed planar annulus (of inner radius $a>0$ and outer radius $b>a$ ) is the set

$$
A[a, b]:=\left\{(x, y) \in \mathbb{R}^{2}: a \leq\|(x, y)\| \leq b\right\} .
$$

Definition 12. A topological space $X$ is called a topological annulus if it is homeomorphic to a planar annulus.

Let $X$ be a topological annulus, and let $\eta: A[1,2] \rightarrow$ $\eta(A[1,2])=X$ be a homeomorphism. As a consequence of the Schoenflies theorem, the set $\eta(\partial A[1,2])$ is independent of the choice of the homeomorphism $\eta$. We call the set $\eta(\partial A[1,2])$ the contour of $X$ and denote it by $\vartheta X$. Clearly, for a topological annulus $X$ embedded in $\mathbb{R}^{2}$, the contour of $X$ coincides with the boundary of $X$. The contour of $X$ consists of two connected components which are closed arcs (the Jordan curves) since they are homeomorphic to $S^{1}$. We call such closed arcs $X_{i}$ and $X_{o}$. For a planarly embedded topological annulus, they could be chosen as the inner and the outer boundaries of the annulus. In such a special case, the bounded component of $\mathbb{R}^{2} \backslash X$ turns out to be an open simply connected set $D=D(X)$ with $\partial D=X_{i}$ and $\operatorname{cl} D=D \cup X_{i}$ homeomorphic to the closed unit disc. On the other hand, in the general setting, speaking of inner and outer boundaries is meaningless, yet we keep this terminology. Finally, we define the interior of $X$ as

$$
\text { int } X:=X \backslash \vartheta X
$$

In the sequel, we use the standard covering projection of $\mathbb{R} \times \mathbb{R}_{0}^{+}$onto $\mathbb{R}^{2} \backslash\{0\}$, defined by the polar coordinates

$$
\Pi(\theta, \rho)=(\rho \cos \theta, \rho \sin \theta) .
$$

In the space $\mathbb{R} \times \mathbb{R}_{0}^{+}$, we consider also the translation

$$
\partial:(\theta, \rho) \longmapsto(\theta+2 \pi, \rho) \text {. }
$$

3.1. Crossing Properties for Annular Regions. Our aim now is to reconsider the results obtained for topological rectangles in Section 2 and adapt them to a form which may be better suited to deal with the new setting of topological annuli. We start with a version of Alexander's lemma which reads as follows.

Lemma 13. Let $X$ be a topological annulus, and let $K_{1}, K_{2}$ be closed subsets of $X$ such that $K_{1} \cap K_{2}$ is connected (possibly empty). Assume that there exist two paths $\gamma_{1}, \gamma_{2}: I \rightarrow X$, with $\gamma_{1}(0), \gamma_{2}(0) \in X_{i}$ and $\gamma_{1}(1), \gamma_{2}(1) \in X_{o}$ such that

$$
\bar{\gamma}_{1} \cap K_{1}=\emptyset, \quad \bar{\gamma}_{2} \cap K_{2}=\emptyset .
$$

Then, there exists a path $\gamma: I \rightarrow X$, with $\gamma(0) \in X_{i}$ and $\gamma(1) \in X_{o}$ such that $\bar{\gamma} \cap\left(K_{1} \cup K_{2}\right)=\emptyset$.

This result is an elementary variant of the version of Alexander's lemma in [29]; see also [38, page 3040] and [37, Corollary 3].

We say that a set $C \subset X$ is essentially embedded in $X$ if the inclusion

$$
i_{C}: C \longrightarrow X, \quad i_{C}(x)=x, \quad \forall x \in C
$$

is not homotopic to a constant map.

The next result is a corollary of the Borsuk separation theorem [41, Theorem 6-47] adapted to our situation. We give a proof, for completeness, following [41].

Lemma 14. Let $X$ be a topological annulus, and let $S \subset X$ be a closed set. Then, $S$ is essentially embedded in $X$ if and only if $S: X_{i}+X_{o}$.

Proof. Up to a homeomorphism defining the annulus $X$, we can assume $X=A[a, b]$ with

$$
0<a<b<1 \text {. }
$$

In this case, $X_{i}=\partial B(0, a)=a S^{1}$ and $X_{o}=\partial B(0, b)=b S^{1}$.

Suppose that $S: X_{i}+X_{o}$, and let $\mathscr{C}(0)$ be the connected component of $\mathbb{R}^{2} \backslash S$ containing the origin. By the assumption, $\mathscr{C}(0) \cup S$ is closed and

$$
B[0, a] \subset \mathscr{C}(0) \cup S \subset B[0, b] .
$$


Assume, by contradiction, that $S$ is not essentially embedded in $X$, and, therefore, the inclusion $i_{S}: S \rightarrow X$ is homotopic in $X$ to a constant map, say $X \ni x \mapsto p$, for all $x \in X$, for a suitable point $p \in X$. From this, it follows immediately that the mapping $f: S \rightarrow S^{1}$ defined by $x \mapsto x /\|x\|$ is homotopic to a constant, which is inessential. Then, by [41, Theorem 4-5], there exists a continuous and inessential extension $\tilde{f}$ of $f$ with $\tilde{f}$ defined on $\mathscr{C}(0) \cup S$. We define now the map $r: E^{2} \rightarrow S^{1}$ by

$$
r(x):= \begin{cases}\tilde{f}(x), & \text { for } x \in \mathscr{C}(0) \cup S \\ \frac{x}{\|x\|}, & \text { for } x \notin \mathscr{C}(0) \cup S\end{cases}
$$

which is continuous. Thus, we are led to a contradiction since $r(\cdot)$ turns out to be a retraction of $E^{2}$ onto $S^{1}$.

Suppose now that $S$ is essentially embedded in $X$, and also assume, by contradiction, that there exists a path $\gamma: I \rightarrow X$ with $\|\gamma(0)\|=a,\|\gamma(1)\|=b$ and that $\gamma(t) \notin S$, for all $t \in I$. Without loss of generality (taking possibly an arc inside $\bar{\gamma}$ ), we can suppose that $\gamma$ is one to one and that $a<\|\gamma(t)\|<b$ for each $t \in] 0,1[$.

Passing to the covering space $\mathscr{H}:=\mathbb{R} \times[a, b]$ of $X=$ $A[a, b]$, we have that the path $\gamma$ lifts to a family of paths $\tilde{\gamma}_{n}$ : $I \rightarrow \mathscr{H}$ with $\tilde{\gamma}_{n}(t)=\tilde{\gamma}_{0}(t)+(2 n \pi, 0)$ and that $\tilde{\gamma}_{n}(t) \cap \Pi^{-1}(S)=$ $\emptyset$, for all $t \in I$ and every $n \in \mathbb{Z}$. By the properties of $\gamma$ we have that $\widetilde{\gamma}_{0}: I \rightarrow \mathscr{H}$ is one to one and, moreover, $\tilde{\gamma}_{0}\left(t^{\prime}\right)-\widetilde{\gamma}_{0}\left(t^{\prime \prime}\right) \notin$ $2 \pi \mathbb{Z}$ for $t^{\prime} \neq t^{\prime \prime}$ (because $\Pi \circ \widetilde{\gamma}_{0}=\gamma$ is one to one). We have thus obtained an $\operatorname{arc} \Gamma_{0}:=\widetilde{\gamma}_{0}(I) \subset \mathscr{H}$ connecting the lines $\rho=a$ and $\rho=b$ and avoiding $\Pi^{-1}(S)$ such that $\Gamma_{0}$ intersects the line $\{\rho=a\}$ exactly at one point and the same happens with respect to $\{\rho=b\}$. Furthermore,

$$
\Gamma_{0} \cap\left((2 \pi n, 0)+\Gamma_{0}\right)=\emptyset, \quad \forall n \in \mathbb{Z} \backslash\{0\} .
$$

Let $\Gamma_{0} \cap\{\rho=a\}=\left\{P_{a}\right\}, \Gamma_{0} \cap\{\rho=b\}=\left\{P_{b}\right\}$, and $\Gamma_{1}:=$ $(2 \pi, 0)+\Gamma_{0}$. Note that $\Gamma_{0} \cap \Gamma_{1}=\emptyset$. Let $\mathcal{J}$ be the Jordan curve obtained by joining (in the counterclockwise sense) the point $P_{a}$ to $(2 \pi, 0)+P_{a}$ along the line $\{\rho=a\}$, the point $(2 \pi, 0)+P_{a}$ to $(2 \pi, 0)+P_{b}$ along $\Gamma_{1}$, the point $(2 \pi, 0)+P_{b}$ to $P_{b}$ along the line $\{\rho=b\}$, and, finally, the point $P_{b}$ to $P_{a}$ along $\Gamma_{0}$. The curve $\mathscr{J}$ is the boundary of an open bounded domain $D$ with $\mathrm{cl} D=$ $D \cup \mathscr{J}$ homeomorphic to the unit square $Q$. Roughly speaking, $\mathrm{cl} D$ is the set of all the points of the strip $\mathscr{H}$ between $\Gamma_{0}$ and $\Gamma_{1}$, with the boundary arcs included. Let $\eta: Q \rightarrow \eta(Q)=\operatorname{cl} D$ be a homeomorphism mapping the left side of $Q$ to $\Gamma_{0}$, the lower side of $Q$ to the segment $\left\{P_{a}+(\theta, 0): \theta \in[0,2 \pi]\right\}$, the right side of $Q$ to $\Gamma_{1}$, and the upper side of $Q$ to the segment $\left\{P_{b}+\right.$ $(\theta, 0): \theta \in[0,2 \pi]\}$. By the construction of the topological rectangle $\mathrm{cl} D$ and since $\Gamma_{0} \cap \Pi^{-1}(S)=\emptyset$, we have that the set

$$
S^{\prime}:=\Pi^{-1}(S) \cap \eta(] 0,1[\times[0,1]) \subset \mathrm{cl} D
$$

is mapped homeomorphically onto $S$ by the covering projection $\Pi$. Now we choose $\varepsilon \in] 0,1 / 2$ [ sufficiently small such that

$$
S^{\prime} \subset \Pi^{-1}(S) \cap \eta([\varepsilon, 1-\varepsilon] \times[0,1]),
$$

and we also introduce the set

$$
B:=\Pi(\eta([\varepsilon, 1-\varepsilon] \times[0,1])) .
$$

By construction, the set $B$ is a topological rectangle contained in $A[a, b]$ and containing the set $S$. The continuous map

$$
\begin{aligned}
& ([\varepsilon, 1-\varepsilon] \times[0,1]) \times[0,1] \ni(z, \lambda) \\
& \quad \longmapsto \Pi\left(\eta\left((1-\lambda) z+\lambda\left(\frac{1}{2}, \frac{1}{2}\right)\right)\right) \subset A[a, b]
\end{aligned}
$$

restricted to $S \times I$ provides a homotopy between the identity $i_{S}$ and a constant map. This contradicts the assumption that $S$ is essentially embedded in $A[a, b]$, and the proof is complete.

A variant of the above proof has been suggested by the referee, and we wish to report it here, since it may be more interesting from a geometrical point of view. The argument is as follows.

Having obtained (as above) $\gamma: I \rightarrow A[a, b]=X$ which is one to one, avoids the set $S$, and intersects the inner and the outer boundaries of $X$ only at its extreme ends $\gamma(0)$ and $\gamma(1)$, we consider a homeomorphism $h$ of the annulus onto itself such that $h(\gamma(I))=\{(x, 0): x \in[a, b]\}, h(\gamma(0))=(a, 0)$, and $h(\gamma(1))=(b, 0)$. Moreover, the segment $[a, b] \times\{0\}$ avoids the set $h(S)$. We lift the segment $[a, b] \times\{0\}$ to sequence of parallel vertical lines $\bigcup_{n \in \mathbb{Z}}\{2 n \pi\} \times[a, b] \subset \mathscr{H}$ which avoid the set $\Pi^{-1}(h(S))$. Now the topological rectangle $\operatorname{cl} D$ of the preceding part of the proof turns out to be just the standard rectangle $[0,2 \pi] \times[a, b]$, and the set $T^{\prime}:=\Pi^{-1}(h(S)) \cap$ (] $0,2 \pi[\times[a, b])$ (which is mapped homeomorphically by $\Pi$ onto $h(S))$ is actually contained in $[\varepsilon, 2 \pi-\varepsilon] \times[a, b]$ for some $\varepsilon>0$ and sufficiently small. Now we conclude precisely as before by showing that $i_{h(S)}$ is homotopic to a constant in the rectangle $\Pi([\varepsilon, 2 \pi-\varepsilon] \times[a, b]) \subset A[a, b]$.

A version of Lemma 10 for a topological annulus reads as follows.

Lemma 15. Let $X$ be a topological annulus, and let $S \subset X$ be a closed set such that $S: X_{i}+X_{o}$. Then, there exists a compact, connected set $C \subset S$ such that $C: X_{i} \nmid X_{o}$ (and, therefore, $C$ is essentially embedded in X).

Proof. The proof follows the same argument of the one of Lemma 10. We give the details for completeness. By Lemma 3, there exists a closed set $C \subset S$ such that $C: X_{i}+X_{o}$, with $C$ minimal with respect to the cutting property. Suppose, by contradiction, that $C$ is not connected, and let $C_{1}, C_{2} \subset C$ be two closed nonempty disjoint sets with $C_{1} \cup C_{2}=C$. Since $C$ is minimal and $C_{1}, C_{2}$ are proper subsets of $C$, there exist two paths $\gamma_{1}, \gamma_{2}$ in $X$ which connect $X_{i}$ to $X_{o}$ such that $\gamma_{i}$ avoids $C_{i}$ (for $i=1,2$ ). Then, by Lemma 13, there exists a path $\gamma: I \rightarrow X$ with $\gamma(0) \in X_{i}$ and $\gamma(1) \in X_{o}$ with $\bar{\gamma} \cap C=\emptyset$, contradicting the assumption that $C: X_{i} \dagger$ $X_{o}$. The continuum $C$ is also essentially embedded in $X$ by Lemma 14.

The result in Lemma 15 has been proved using a minimality argument. In some cases, the minimality of the set $C$ may be useful for the proof of some topological properties of the continuum. An example in this direction is given in the next lemma. 
Lemma 16. Let $X$ be a topological annulus, and let $C \subset X$ be a compact connected set which is minimal with respect to the property of cutting all the paths in $X$ from $X_{i}$ to $X_{o}$. Let $f: C \rightarrow \mathbb{R}$ be a nonconstant continuous function. Then, for every $k \in] \min f(C), \max f(C)$ [ there exist at least two points $w, z \in C$ with $w \neq z$ such that $f(w)=f(z)=k$.

Proof. Without loss of generality, we can assume that $k=$ 0 and $f$ changes sign on $C$. The existence of at least a zero for $\left.f\right|_{C}$ follows from Bolzano's theorem. Suppose, by contradiction, that there is only one point say $z \in C$ such that $f(z)=0$. Consider the two nonempty compact sets $K_{1}:=\{x \in C: f(x) \leq 0\}$ and $K_{2}:=\{x \in C: f(x) \geq 0\}$. By the assumption, we have that $\{z\}=K_{1} \cap K_{2}$ and $K_{1} \neq C$ as well as $K_{2} \neq C$. By the assumption of minimality of $C$, it follows that there exists a path $\gamma_{1}$ connecting $X_{i}$ to $X_{o}$ in $X$ and avoiding $K_{1}$ and, similarly, there exists a path $\gamma_{2}$ connecting $X_{i}$ to $X_{o}$ in $X$ and avoiding $K_{2}$. From Lemma 13 we know that there exists a path $\gamma$ in $X$ connecting $X_{i}$ to $X_{o}$ and avoiding $C=K_{1} \cup K_{2}$. This contradicts the cutting property of $C$.

We observe that the existence of two solutions is not guaranteed if the minimality of the set $C$ is not assumed (see [42, Example 2.8]).

In the case of topological rectangles, there is a complete symmetry between the fact that a set cuts the paths between a given pair of opposite sides or it cuts the paths connecting a complementary pair of opposite sides. Thus, once we have achieved a result like Lemma 11, also its dual version, involving the other pair of sides, is guaranteed. In the case of topological annuli, the situation is different. We have just proved a result which expresses the fact that a compact set which crosses all the paths from the inner to the outer boundary must contain a continuum which nontrivially winds around the annulus. A dual result should express the fact that if a compact set intersects all the nontrivial loops of the annulus, then it must contain a continuum joining the inner and the outer boundaries of the annulus. This is precisely the content of the next lemma. To this end, we first recall some basic facts from homotopy theory. Let $\omega: I \rightarrow X$ be a loop, which is a continuous path such that $\omega(0)=\omega(1)$. We say that $\omega$ is (homotopically) trivial in $X$ if it is homotopic in $X$ to the constant loop $e_{x_{0}}: I \rightarrow x_{0}$ with $x_{0}=\omega(0)=\omega(1)$. Since a loop in $X$ (up to a change in the parameter) may be also seen as a continuous map $\omega: S^{1} \rightarrow X$, triviality of $\omega$ can be also expressed by the fact that there is a continuous extension $\alpha: E^{2} \rightarrow X$ with $\alpha_{S^{1}}=\omega$. We say that a loop $\omega$ in $X$ is nontrivial if it is not homotopically trivial in $X$. Then we have the following.

Lemma 17. Let $X$ be a topological annulus, and let $S \subset X$ be a closed set such that $S \cap \bar{\omega} \neq \emptyset$ for each nontrivial loop $\omega$ in $X$. Then, there exists a compact, connected set $C \subset S$ such that

$$
C \cap X_{i} \neq \emptyset \neq C \cap X_{o} \text {. }
$$

Proof. Without loss of generality (up to a homeomorphism), we suppose that $X=A[a, b]$ with $0<a<b$, so that $X$ has inner boundary $X_{i}=a S^{1}$ and outer boundary $X_{o}=b S^{1}$. By the assumption of crossing the nontrivial loops, we know that $S_{i}:=S \cap X_{i} \neq \emptyset$ and also $S_{o}:=S \cap X_{o} \neq \emptyset$.

Suppose, by contradiction, that $S$ does not contain any compact connected set $C$ satisfying (21). Then, by the Kuratowski-Whyburn lemma [13,40], it follows that $S$ splits as the disjoint union of two compact sets $S^{\prime}, S^{\prime \prime}$ with $S^{\prime} \supset S_{i}$ and $S^{\prime \prime} \supset S_{o}$. We pass now to the covering space $\mathscr{H}:=\mathbb{R} \times[a, b]$ of $X=A[a, b]$ and consider the closed subsets of $\mathscr{H}$,

$$
W^{\prime}:=\Pi^{-1}\left(S^{\prime}\right) \cup(\mathbb{R} \times\{a\}), \quad W^{\prime \prime}:=\Pi^{-1}\left(S^{\prime \prime}\right) \cup(\mathbb{R} \times\{b\}) .
$$

By definition, $\Pi\left(W^{\prime} \cup W^{\prime \prime}\right) \supset S$. Moreover, $W^{\prime} \cap W^{\prime \prime}=\emptyset$, and both such sets are invariant with respect to the translation $D$ defined in (10). We define

$$
\begin{aligned}
\delta & :=\operatorname{dist}\left(W^{\prime}, W^{\prime \prime}\right) \\
& =\inf \left\{\left\|w^{\prime}-w^{\prime \prime}\right\|: w^{\prime} \in W^{\prime}, w^{\prime \prime} \in W^{\prime \prime}\right\} .
\end{aligned}
$$

It is clear that $\delta>0$, and it is actually a minimum (this follows from a standard compactness argument, using the periodicity). Then, we define the two closed $\varepsilon$-tubular neighborhoods of $W^{\prime}$ and $W^{\prime \prime}$ as $W^{\prime}[\varepsilon]:=\{y=(\theta, \rho) \epsilon$ $\left.\mathscr{H}: \operatorname{dist}\left(y, W^{\prime}\right) \leq \varepsilon\right\}$ and $W^{\prime \prime}[\varepsilon]:=\{y=(\theta, \rho) \in \mathscr{H}:$ $\left.\operatorname{dist}\left(y, W^{\prime \prime}\right) \leq \varepsilon\right\}$, for

$$
0<\varepsilon \leq \frac{\delta}{3}
$$

We modify now a pigeonhole argument used in the proof of Theorem 1 in [10] as follows. Let us fix a positive integer $N>$ $(b-a) / \varepsilon$ and consider the rectangle

$$
\mathscr{R}:=[0,2 N \pi] \times[a, b] .
$$

The sets

$$
K_{1}:=W^{\prime}[\varepsilon] \cap \mathscr{R}, \quad K_{2}:=W^{\prime \prime}[\varepsilon] \cap \mathscr{R}
$$

are closed and disjoint. The lower edge $[0,2 N \pi] \times\{a\}$ is the image of a path connecting the left to the right side of $\mathscr{R}$ and avoiding the set $K_{2}$. Similarly, via the upper edge $[0,2 N \pi] \times$ $\{b\}$ we find a path connecting the left to the right side of $\mathscr{R}$ and avoiding the set $K_{1}$. Alexander's lemma (Lemma 8) guarantees the existence of a path $\gamma=(\theta(t), \rho(t)): I \rightarrow \mathscr{R}$ with $\theta(0)=0$ and $\theta(1)=2 N \pi$, such that $\gamma(t) \notin K_{1} \cup K_{2}$ for all $t \in I$. For each $i=0, \ldots, N$, let

$$
t_{i}:=\min \{t \in I: \theta(t)=2 i \pi\},
$$

so that

$$
0=t_{0}<t_{1}<\cdots<t_{N} \leq 1
$$

and the $N+1$-tuple of points $\left(\rho\left(t_{0}\right), \rho\left(t_{1}\right), \ldots, \rho\left(t_{N}\right)\right)$ in $] a, b[$ is well defined. Clearly, by the choice of $N$ such that $N \varepsilon>$ $(b-a)$, there exists at least a pair of points $\left(t_{j}, t_{k}\right)$ with $j<k$ such that $\left|\rho\left(t_{j}\right)-\rho\left(t_{k}\right)\right|<\varepsilon$. Since

$$
\min _{t \in[0,1]}\left\{\operatorname{dist}\left(\gamma(t), W^{\prime} \cup W^{\prime \prime}\right)\right\}>\varepsilon,
$$


we conclude that the segment joining the points $\left(2 k \pi, \rho\left(t_{j}\right)\right)$ and $\left(2 k \pi, \rho\left(t_{k}\right)\right)$ does not intersect the set $W^{\prime} \cup W^{\prime \prime}$ (of course, such statement is trivial if $\left.\rho\left(t_{j}\right)=\rho\left(t_{k}\right)\right)$. We can now define the path

$$
\begin{aligned}
& \tilde{\omega}(s) \\
& := \begin{cases}\gamma\left(t_{j}+2 s\left(t_{k}-t_{j}\right)\right), & \text { for } 0 \leq s \leq \frac{1}{2}, \\
\left(2 k \pi, \rho\left(t_{k}\right)+(2 s-1)\left(\rho\left(t_{j}\right)-\rho\left(t_{k}\right)\right)\right), & \text { for } \frac{1}{2} \leq s \leq 1,\end{cases}
\end{aligned}
$$

which takes values in $\mathscr{R} \backslash\left(W^{\prime} \cup W^{\prime \prime}\right)$ and is such that

$$
\widetilde{\omega}(0)=\left(2 j \pi, \rho\left(t_{j}\right)\right), \quad \widetilde{\omega}(1)=\left(2 k \pi, \rho\left(t_{j}\right)\right) .
$$

Hence, the projection $\omega:=\Pi \circ \widetilde{\omega}: I \rightarrow X$ is a nontrivial loop in $X$ (in fact, it corresponds to $k-j \in \mathbb{Z} \backslash\{0\}$ in the fundamental group of $X)$ and, by construction, $\omega(t) \notin$ $S$, for all $t \in I$. This contradicts the hypothesis, and hence, the conclusion follows.

Our last result in this section can be seen as a continuous version of the no-tie theorem for the Hex game on the annulus. See [10] for a discrete version of this result.

Lemma 18. Let $X$ be a topological annulus, and let $C_{1}, C_{2} \subset X$ be closed connected sets such that

$$
C_{1} \cap X_{i} \neq \emptyset \neq C_{1} \cap X_{o}
$$

$C_{2}$ is essentially embedded in $X$.

Then, $C_{1} \cap C_{2} \neq \emptyset$.

Proof. Assume, by contradiction, that $C_{1} \cap C_{2}=\emptyset$, and let $\operatorname{dist}\left(C_{1}, C_{2}\right)=\delta>0$. In a $\delta / 2$-neighborhood of $C_{1}$, we can find the image of a path $\gamma: I \rightarrow X$ with $\gamma(0) \in X_{i}$ and $\gamma(1) \in$ $X_{o}$. By construction, $\gamma(t) \notin C_{2}$, for all $t \in I$. This proves that it is not true that $C_{2}: X_{i}+X_{o}$, and therefore (by Lemma 14) $C_{2}$ is not essentially embedded in $X$, thus contradicting one of our assumptions.

\section{A Crossing Lemma for Invariant Sets}

Let $X$ be a topological space, and let $h: X \rightarrow X$ be a homeomorphism. Our first result is a version of Lemma 3 for $h$-invariant sets. Indeed, we have the following.

Lemma 19. Let $A, B \subset X$ be two nonempty disjoint sets which are connected by at least one path in $X$. Let $S \subset X$ be a closed set which satisfies $S: A+B$ and is invariant for $h$. Then, there exists a nonempty, closed set $C \subset S$ which is minimal with respect to the property of cutting the paths between $A$ and $B$ and is invariant for $h$.

Proof. Let $\mathscr{F}$ be the set of all the nonempty closed subsets $F$ of $S$ such that $F: A+B$ and $h(F)=F$, with the elements of $\mathscr{F}$ ordered by inclusion. Let $\left(F_{\alpha}\right)_{\alpha \in J}$ be a totally ordered subset of $\mathscr{F}$, and define $F^{*}:=\bigcap_{\alpha \in J} F_{\alpha}$. From $h\left(F_{\alpha}\right)=F_{\alpha}$ for all $\alpha \in J$, it follows that $h\left(F^{*}\right)=F^{*}$. The proof that $F^{*}: A+B$ is the same as that of Lemma 3. Thus, we obtain $F^{*} \in \mathscr{F}$, and the conclusion follows from Zorn's lemma.

Our goal now is to develop a result analog of Lemma 10 in the frame of $h$-invariant sets. Note that if in Lemma 10 we have a continuum $C: X_{l}^{-} \nmid X_{r}^{-}$which is also $h$-invariant, then by Lemma 11 we also have an $h$-invariant continuum intersecting $X_{b}^{+}$and $X_{t}^{+}$.

In order to fix the ideas and before moving to the class of generalized and oriented rectangles, we consider for a moment a planar rectangle $\mathscr{R}=[a, b] \times[c, d]$ oriented in the standard manner and suppose that $h: \mathscr{R} \rightarrow \mathscr{R}$ is a homeomorphism. Let $S \subset \mathscr{R}$ also be a compact set which intersects all the paths in $\mathscr{R}$ joining $\mathscr{R}_{l}^{-}$to $\mathscr{R}_{r}^{-}$such that $h(S)=S$. We are looking for the existence of a continuum $C \subset S$ with $C: \mathscr{R}_{l}^{-}+\mathscr{R}_{r}^{-}$and $h(C)=C$. It is not difficult to see that, in general, the answer is negative, as shown by the following elementary example.

Example 20. Let $\mathscr{R}=[-2,2] \times[0,1]$ and $S=\{( \pm 1, y)$ : $y \in[0,1]\}$. Clearly, $S$ has the property of intersecting all the paths in $\mathscr{R}$ joining the left edge to the right edge. Moreover, consistently with Lemma $10, S$ contains two continua $\{-1\} \times$ $[0,1]$ and $\{1\} \times[0,1]$ connecting the lower and the upper sides of $\mathscr{R}$. However, if we take as a homeomorphism $h(x, y)=$ $(-x, y)$, that is, the symmetry with respect to the $y$-axis, we have that $h(S)=S$, but there is no connected subset of $S$ which is invariant under $h$.

Observe that in Example 20 the set $S$ cannot be split as the union of two disjoint closed (nonempty) subsets which are invariant. This property is also expressed by saying that $S$, (although not connected) is invariantly connected (see [43, Definition 4.2]). If we are allowed to replace the word "connected" with "invariantly connected," we can get a full extension of Lemma 10, as follows.

Lemma 21. Let $\widetilde{X}=\left(X, X^{-}\right)$be an oriented rectangle, let $h$ : $X \rightarrow X$ be a homeomorphism, and let $S \subset X$ be a closed set such that

$$
S: X_{l}^{-}+X_{r}^{-}, \quad h(S)=S \text {. }
$$

Then, there exists a compact, invariantly connected set $C \subset S$ such that

$$
C: X_{l}^{-}+X_{r}^{-}
$$

Proof. By Lemma 19 there exists a closed set $C \subset S$ such that $C: X_{l}^{-}+X_{r}^{-}$, with $C$ invariant for $h$ and minimal with respect to the cutting property. Suppose, by contradiction, that $C$ is not invariantly connected, and let $C_{1}, C_{2} \subset C$ be two closed nonempty disjoint sets with $C_{1} \cup C_{2}=C$ and $h\left(C_{i}\right)=C_{i}$ for $i=1,2$. Now we conclude as in the proof of Lemma 10 . Indeed, since $C$ is minimal and $C_{1}, C_{2}$ are proper subsets of $C$, there exist two paths $\gamma_{1}, \gamma_{2}$ in $X$ which connect $X_{l}^{-}$to $X_{r}^{-}$, such that $\gamma_{i}$ avoids $C_{i}$ (for $\left.i=1,2\right)$. Then, by Lemma 8 , there exists a path $\gamma: I \rightarrow X$ with $\gamma(0) \in X_{l}^{-}$and $\gamma(1) \in X_{r}^{-}$ with $\bar{\gamma} \cap C=\emptyset$, contradicting the assumption that $C: X_{l}^{-}+$ $X_{r}^{-}$. 
In order to have the connectedness of the set $C$, we propose a partial extension of Lemma 10 by adding a further assumption on $h$ which prevents the possibility of a situation like the one described in Example 20. For simplicity in the exposition, we confine ourselves to the case of a planar rectangle $\mathscr{R}=[a, b] \times[c, d]$. Here, we do not require $h$ to be a homeomorphism.

Lemma 22 ( $h$-invariant crossing lemma). Let $\mathscr{R}=[a, b] \times$ $[c, d]$, and let $h: \mathscr{R} \rightarrow \mathscr{R}$ be a continuous map such that

$$
h\left(\mathscr{R}_{b}^{+}\right) \subset \mathscr{R}_{b}^{+}, \quad h\left(\mathscr{R}_{t}^{+}\right) \subset \mathscr{R}_{t}^{+} .
$$

Suppose that there exists a path $\sigma: I \rightarrow \mathscr{R}$ with $\sigma(0) \in \mathscr{R}_{l}^{-}$ and $\sigma(1) \in \mathscr{R}_{r}^{-}$such that $\forall t \in I, \exists s \geq t: h(\sigma(t))=\sigma(s)$.

Assume that there exists a compact set $S \subset \mathscr{R}$ which cuts the paths between $\mathscr{R}_{l}^{-}$and $\mathscr{R}_{r}^{-}$and satisfies

$$
h(S) \subset S \text {. }
$$

Then, there exists a compact connected set $C \subset S$ such that

(i) $h(C)=C$,

(ii) $C \cap \mathscr{R}_{b}^{+} \neq \emptyset \neq C \cap \mathscr{R}_{t}^{+}$,

(iii) $C$ cuts the paths between $\mathscr{R}_{l}^{-}$and $\mathscr{R}_{r}^{-}$.

Proof. As a first step, we apply Lemmas 10 and 11 which guarantee the existence of a continuum contained in $S$ which joins the lower and the upper sides of the rectangle and cuts the paths from the left to the right side in $\mathscr{R}$. We call such a continuum $C^{0}$. Note that $C^{0}$ is not necessarily invariant.

In order to obtain an invariant set, we define a sequence of continua by naming

$$
C^{i+1}=h\left(C^{i}\right), \quad \forall i \geq 0 .
$$

Since $h(S) \subset S$, we also know that $C^{i} \subset S$, for all $i \geq 0$. By the cutting property of $C^{0}$, we have that

$$
C^{0} \cap \bar{\sigma} \neq \emptyset
$$

so that there exists $t_{0} \in I$ with $\sigma\left(t_{0}\right) \in C^{0}$. Clearly, $h\left(\sigma\left(t_{0}\right)\right) \in$ $C^{1}$. On the other hand, by the hypothesis on $\sigma$, there exists $t_{1} \in I$ such that $t_{1} \geq t_{0}$ and $h\left(\sigma\left(t_{0}\right)\right)=\sigma\left(t_{1}\right) \in C^{1}$. Proceeding by induction and using step by step the hypothesis on $\sigma$, we obtain a monotone sequence $t_{0} \leq t_{1} \leq \cdots t_{i} \leq t_{i+1} \leq \cdots$ in $[0,1]$ such that $\sigma\left(t_{i}\right) \in C^{i}$, for all $i \geq 0$. Let $t_{i} \nearrow t^{*} \in[0,1]$. Then, passing to the limit in $\sigma(\cdot)$ and $h \circ \sigma$, we obtain

$$
h\left(\sigma\left(t^{*}\right)\right)=\sigma\left(t^{*}\right) \in \operatorname{Li} C^{i}
$$

where $\operatorname{Li} C^{i}$ is the set of all the points $x$ such that for every open neighborhood $U$ of $x$ it holds that $U \cap C^{i} \neq \emptyset$ for each $i$ large enough. Therefore, by a classical result from [40, Theorem 6, Chapter 5, Section 47,II], we have that

$$
C:=\operatorname{Ls} C^{i}
$$

is a nonempty continuum (where Ls $C^{i}$ is the set of all the points $x$ such that for every open neighborhood $U$ of $x$ it holds that $U \cap C^{i} \neq \emptyset$ for infinitely many $i$ ). Recall that $z \in C$ if and only if there exists a sequence $z_{k}$ with $z_{k} \in C^{i_{k}}$ for $\left(i_{k}\right)_{k}$ an increasing sequence of indexes such that $z_{k} \rightarrow z$. Then, $h\left(z_{k}\right) \rightarrow h(z)$, with $h\left(z_{k}\right) \in C^{1+i_{k}}$, and, therefore, $h(z) \in C$. Thus, we have proved that $h(C) \subset C$. Conversely, for $z \in C$ and $\left(z_{k}\right)_{k} \rightarrow z$, as above, let us fix $k \geq 2$, and, from $z_{k} \in$ $C^{i_{k}}=h\left(C^{-1+i_{k}}\right)$, let us take $y_{k} \in C^{-1+i_{k}}$ such that $h\left(y_{k}\right)=z_{k}$. By compactness, $\left(y_{k}\right)_{k}$ has a convergent subsequence. Let us set $\left(y_{k_{n}}\right)_{n} \rightarrow w$. By definition, we have that $w \in C$. On the other hand, $h(w)=\lim h\left(y_{k_{n}}\right)=\lim z_{k_{n}}=z$. This proves that $h(C)=C$.

Since we know that $C^{0} \cap \mathscr{R}_{b}^{+} \neq \emptyset$, we have

$$
\emptyset \neq h\left(C^{0} \cap \mathscr{R}_{b}^{+}\right) \subset h\left(C^{0}\right) \cap h\left(\mathscr{R}_{b}^{+}\right) \subset C^{1} \cap \mathscr{R}_{b}^{+} .
$$

From this, by induction, we obtain that

$$
C^{i} \cap \mathscr{R}_{b}^{+} \neq \emptyset, \quad \forall i \geq 0
$$

and then, by compactness, we conclude that $C \cap \mathscr{R}_{b}^{+} \neq \emptyset$. The fact that $C \cap \mathscr{R}_{t}^{+} \neq \emptyset$ is proved in the same manner. Having proved that $C$ is a continuum intersecting the horizontal edges of the rectangle, we conclude that it cuts all the paths between $\mathscr{R}_{l}^{-}$and $\mathscr{R}_{r}^{-}$.

Remark 23. Observe that the same result holds true (with an obvious modification in the proof) if we replace condition (35) with

$$
h\left(\mathscr{R}_{b}^{+}\right) \subset \mathscr{R}_{t}^{+}, \quad h\left(\mathscr{R}_{t}^{+}\right) \subset \mathscr{R}_{b}^{+}
$$

Remark 24. By the assumption $h\left(\mathscr{R}_{b}^{+}\right) \subset \mathscr{R}_{b}^{+}$in (35), we have that

$$
h(x, c)=(f(x), c), \quad \forall x \in[a, b],
$$

where $f:[a, b] \rightarrow[a, b]$ is a suitable continuous function. It easily follows that if $f$ is monotone nondecreasing, then the path $\sigma(t):=(a+t(b-a), c)$ satisfies the hypothesis of Lemma 22. A similar observation holds for $\mathscr{R}_{t}^{+}$. Hence, we easily obtain the following.

Corollary 25. Let $\mathscr{R}$ be as in Lemma 22, and let $h: \mathscr{R} \rightarrow \mathscr{R}$ be a continuous map satisfying (35), and suppose also that at least one between $\left.h\right|_{\mathscr{R}_{b}^{+}}$and $\left.h\right|_{\mathscr{R}_{t}^{+}}$is monotone nondecreasing. Assume that there exists a compact set $S \subset \mathscr{R}$ which cuts the paths between $\mathscr{R}_{l}^{-}$and $\mathscr{R}_{r}^{-}$and satisfies $h(S) \subset S$. Then, the same conclusion of Lemma 22 holds.

Notice that in Example 20 the function $h$ is decreasing along both horizontal edges of the rectangle.

A trivial case of a continuous map which is monotone nondecreasing along the horizontal lines is the identity. In such a case, Corollary 25 reduces to the crossing lemma in Section 2.

A useful property of the continua connecting two compact disjoint sets is the minimality, often named as irreducibility [13]. Indeed, a stronger version of Lemma 10 holds, guaranteeing the existence of compact connected set $C \subset S$ 
which is irreducible between $X_{l}^{-}$and $X_{r}^{-}$(see [13, Proposition 3]).

Then, as a next step, we look for the existence of irreducible invariant continua in this new setting. Such a result, indeed, is of general nature and independent of the fact that in the present paper we consider only the case of planar continua.

Lemma 26. Let $X$ be a compact Hausdorff space, and let $h$ : $X \rightarrow X$ be a homeomorphism. Assume that $A, B \subset X$ are closed disjoint sets which are invariant for $h$, and let $C \subset X$ be a continuum such that

$$
h(C)=C, \quad C \cap A \neq \emptyset \neq C \cap B .
$$

Then, there exists $\mathscr{E} \subset C$ satisfying (45) and minimal with respect to such property.

Proof. Let $\mathscr{F}$ be the family of all the continua $\mathscr{C} \subset C$ which are invariant for the homeomorphism $h$ and intersect both $A$ and $B$, with $\mathscr{F}$ ordered by inclusion. The family $\mathscr{F}$ is nonempty since $C \in \mathscr{F}$. The existence of a minimal subcontinuum of $C$ satisfying (45) will be ensured by Zorn's lemma.

Given any chain $\left(\mathscr{C}_{j}\right)_{j \in \mathscr{L}}$ in $\mathscr{F}$, we observe that $\bigcap_{j \in \mathscr{J}} \mathscr{C}_{j} \neq \emptyset$ for every finite subset of indices $\mathscr{J} \subset \mathscr{L}$. Hence, by compactness, $C^{*}:=\bigcap_{j \in \mathscr{L}} C_{j}$ is nonempty. Moreover $C^{*}$ is compact and intersects both $A$ and $B$. Using the fact that $h$ is a homeomorphism, we obtain the invariance of $C^{*}$. Thus, if we prove that $C^{*}$ is connected, we have that $C^{*} \in \mathscr{F}$, and Zorn's lemma allows to conclude the proof.

Assume, by contradiction, that $C^{*}$ is not connected. Then, there exist $C^{\prime}, C^{\prime \prime}$ nonempty compact sets such that $C^{*}=$ $C^{\prime} \cup C^{\prime \prime}$ and $C^{\prime} \cap C^{\prime \prime}=\emptyset$. Then, there are also two open disjoint sets $A^{\prime}, A^{\prime \prime}$ with $A^{\prime} \supset C^{\prime}$ and $A^{\prime \prime} \supset C^{\prime \prime}$.

We claim that there exists an index $j^{*}$ such that the set $C_{j^{*}} \subset A^{\prime} \cup A^{\prime \prime}$. Otherwise, it would happen that $C_{j} \not \subset A^{\prime} \cup$ $A^{\prime \prime}$ which implies that $D_{j}:=C_{j}-\left(A^{\prime} \cup A^{\prime \prime}\right) \neq \emptyset, \forall j \in \mathscr{L}$. The family $\left(D_{j}\right)_{j \in \mathscr{L}}$ is a family of closed sets with the finiteintersection property, and, therefore, we obtain

$$
\exists \widehat{x} \in \bigcap_{j \in \mathscr{L}} D_{j} \subset X-\left(A^{\prime} \cup A^{\prime \prime}\right) .
$$

This is a contradiction to $\bigcap_{j \in \mathscr{L}} D_{j} \subset \bigcap_{j \in \mathscr{L}} C_{j}=C^{*} \subset A^{\prime} \cup$ $A^{\prime \prime}$.

To conclude, we observe that $C^{*} \subset C_{j^{*}} \subset A^{\prime} \cup A^{\prime \prime}$ with $C_{j^{*}}$ a connected set. Since $C^{*}=C^{\prime} \cup C^{\prime \prime}$ with $C^{\prime} \subset A^{\prime}$ and $C^{\prime \prime} \subset A^{\prime \prime}$, we have that $C_{j^{*}} \cap A^{\prime} \neq \emptyset$ and $C_{j^{*}} \cap A^{\prime \prime} \neq \emptyset$, which contradicts the fact that $C_{j^{*}}$ is connected (in fact, $A^{\prime}$ and $A^{\prime \prime}$ are disjoint).

\section{Applications}

We conclude this paper by presenting a possible application of the above-described classical separation results to an example which is inspired by a model arising from the theory of fluid mixing previously considered by Kennedy and Yorke in [17].
Following [17, Section 2], we consider a planar map $\Phi$ : $\mathbb{R}^{2} \rightarrow \mathbb{R}^{2}$ which is the composition of a squeeze map $J_{\lambda}$ and a rotation map $R_{\theta_{0}}$. In [17], these two maps are defined as follows:

$$
J_{\lambda}(x, y)=\left(\lambda x, \frac{y}{\lambda}\right), \quad \text { with } \lambda>1 .
$$

In order to define the map $R_{\theta_{0}}$ we pass to the polar coordinates $(\theta, \rho)$ and require that $R_{\theta_{0}}$ is a counterclockwise rotation which leaves all the concentric circumferences $\rho>0$ invariant and keeps all the points of the plane with $\rho \geq 1$ still, and, moreover,

$$
\lim _{\rho \rightarrow 0^{+}} \Theta(\theta, \rho)=\theta_{0}>0,
$$

where $\Theta(\theta, \rho)$ is the angular displacement performed by $R_{\theta_{0}}$ on the point $(\rho \cos \theta, \rho \sin \theta)$.

In [17], under the hypotheses that $R_{\theta_{0}}$ is a diffeomorphism and that $\theta_{0}>\pi / 2$, with $\theta_{0}$ not an odd multiple of $\pi / 2$, the authors prove the existence of a Smale horseshoe if $\lambda>0$ is sufficiently large. In particular, there exists an invariant Cantor set on which the map $F:=R_{\theta_{0}} \circ J_{\lambda}$ is conjugate to an $m$-shift.

We are going to prove a result which, although not so sharp like that in [17], makes use of weaker conditions. To be more specific, from now on, the following assumptions are made.

Let $J: \mathbb{R}^{2} \rightarrow \mathbb{R}^{2}$ be a continuous map with

$$
J(x, y)=\left(J_{1}(x, y), J_{2}(x, y)\right)
$$

which satisfies the following properties:

(i) $J(0)=0, J(z) \neq 0$ for $z \neq 0$, and $J\left(Q_{i}\right) \subset Q_{i}$ for $i=1, \ldots, 4$, where with $Q_{i}$ we denote the $i$ th closed quadrant of the plane,

(ii) $J_{2}(x, 0)=0$ and $J_{1}(x, 0)=a(x) x$, with $a(x)>1$ for $x \neq 0$,

(iii) $J_{1}(0, y)=0$ and $J_{2}(0, y)=b(y) y$, with $0<b(y)<1$ for $y \neq 0$.

Plainly speaking, $J$ leaves the quadrants invariant and moves the points of the $x$-axis away from the origin, while it pushes the points of the $y$-axis toward the origin. In the sequel it will be convenient to express the map $J$ (restricted to $\mathbb{R}^{2} \backslash\{0\}$ ) via its lifting to the covering space $\mathbb{R} \times \mathbb{R}_{0}^{+}$as

$$
\begin{gathered}
\tilde{J}:(\theta, \rho) \longmapsto\left(\theta^{\prime}, \rho^{\prime}\right), \\
\theta^{\prime}=\theta+\Theta_{J}(\theta, \rho), \quad \rho^{\prime}=\mathscr{R}_{J}(\theta, \rho),
\end{gathered}
$$

with $\Theta_{J}$ and $\mathscr{R}_{J}$ continuous functions which are $2 \pi$-periodic in the $\theta$-variable. Note that the assumption $J\left(Q_{i}\right) \subset Q_{i}$, for $i=1, \ldots, 4$, reflects the fact that

$$
\left|\Theta_{J}(\theta, \rho)\right| \leq \frac{\pi}{2} .
$$

As a second map, we consider a continuous counterclockwise rotation $R$ around the origin such that $R(0)=0$ and $R(z) \neq 0$ 
for $z \neq 0$. We also express $R$ (restricted to $\mathbb{R}^{2} \backslash\{0\}$ ) by means of polar coordinates through its lifting

$$
\begin{gathered}
\widetilde{R}:(\theta, \rho) \longmapsto\left(\theta^{\prime}, \rho^{\prime}\right), \\
\theta^{\prime}=\theta+\Theta_{R}(\theta, \rho), \quad \rho^{\prime}=\mathscr{R}_{R}(\theta, \rho),
\end{gathered}
$$

with $\Theta_{R}$ and $\mathscr{R}_{R}$ continuous functions which are $2 \pi$-periodic in the $\theta$-variable, and assume the following conditions.

There exist $\left.r_{0} \in\right] 0,1\left[\right.$ and $\theta_{0}$ such that

(i) $\Theta_{R}\left(\theta, r_{0}\right) \geq \theta_{0}, \Theta_{R}(\theta, 1)=0$, for all $\theta \in \mathbb{R}$,

(ii) $\mathscr{R}_{R}(\theta, \rho)=\rho$, for all $\theta \in \mathbb{R}$ and $\rho \in\left[r_{0}, 1\right]$.

According to the above hypotheses, the map $R$ leaves the circumferences of center the origin and radius $\rho \in\left[r_{0}, 1\right]$ invariant. Moreover, the points with $\rho=r_{0}$ are rotated in the counterclockwise sense by an angle larger or equal to $\theta_{0}$, while the points with $\rho=1$ are kept still.

Under the previous assumptions on $J$ and $R$, the following result holds.

Theorem 27. Let $R$ be a homeomorphism of the annulus $\mathscr{A}:=$ $A\left[r_{0}, 1\right]$ onto itself, and suppose also that $\theta_{0}>2 \pi+\pi / 2$. Then, the map $\Psi:=J \circ R$ has at least four fixed points in the interior of the annulus. Such result is stable with respect to small continuous perturbations of the map $\Psi$.

Proof. Let us denote by $\mathscr{A}_{i}$ and $\mathscr{A}_{0}$ the inner and outer boundaries of $\mathscr{A}$. We also restrict the map $\Psi$ to the annulus $\mathscr{A}$ and consider its lifting $\widetilde{\Psi}$ the covering space $\mathbb{R} \times\left[r_{0}, 1\right]$ as

$$
\begin{gathered}
\widetilde{\Psi}(\theta, \rho) \longmapsto\left(\theta^{\prime \prime}, \rho^{\prime \prime}\right), \\
\theta^{\prime \prime}=\theta+\Theta_{\Psi}(\theta, \rho), \quad \rho^{\prime \prime}=\mathscr{R}_{\Psi}(\theta, \rho),
\end{gathered}
$$

with $\Theta_{\Psi}$ and $\mathscr{R}_{\Psi}$ continuous functions which are $2 \pi$-periodic in the $\theta$-variable. By the above positions for $\widetilde{J}$ and $\widetilde{R}$ we have that

$$
\begin{aligned}
& \theta^{\prime \prime}=\theta^{\prime}+\Theta_{J}\left(\theta^{\prime}, \rho^{\prime}\right), \\
& \text { with } \theta^{\prime}=\theta+\Theta_{R}(\theta, \rho), \quad \rho^{\prime}=\mathscr{R}_{R}(\theta, \rho) .
\end{aligned}
$$

We also introduce a set $S \subset \mathscr{A}$ defined as

$$
S:=\Pi\left(\left\{(\theta, \rho): \Theta_{\Psi}(\theta, \rho)=2 \pi\right\}\right),
$$

where $\Pi$ is the standard covering projection associated with the polar coordinates. The set $S$ is a compact subset of the annulus consisting of the points which are rotated by an angle of exactly $2 \pi$ under the action of $\Psi$.

Suppose that $\gamma: I \rightarrow \mathscr{A}$ is a path with $\gamma(0) \in \mathscr{A}_{i}$ and $\gamma(1) \in \mathscr{A}_{0}$. We express the points of $\gamma(t)$ in polar coordinates as

$$
\gamma(t)=(\rho(t) \cos \theta(t), \rho(t) \sin \theta(t))
$$

and consider the angular displacement for the map $R$ along the points of $\gamma(t)$, using the function

$$
\omega_{\gamma}(t): I \ni t \longmapsto \Theta_{R}(\theta(t), \rho(t)) .
$$

By the assumptions, $\omega_{\gamma}(0)>2 \pi+\pi / 2$ and $\omega_{\gamma}(1)=0$. Now we apply the map $J$ to the points of $R(\gamma(t))$. Using condition (51), we have that the angular displacement $\Theta_{\Psi}$ along the curve $\gamma(t)$ can be expressed as

$$
\Theta_{\Psi}(\theta(t), \rho(t))=\omega_{\gamma}(t)+\Delta(t),
$$

where $\Delta(t)$ is a continuous function satisfying

$$
|\Delta(t)| \leq \frac{\pi}{2}, \quad \forall t \in[0,1] .
$$

Recalling the properties of $\omega_{\gamma}$, we find

$$
\begin{gathered}
\Theta_{\Psi}(\theta(0), \rho(0))>2 \pi, \\
\Theta_{\Psi}(\theta(1), \rho(1)) \leq \frac{\pi}{2}<2 \pi,
\end{gathered}
$$

and, therefore, by the continuity of $\gamma$, we can conclude that $\bar{\gamma} \cap S \neq \emptyset$. Now we can apply Lemma 15 which ensures the existence of a compact connected set $C \subset S$ which is essentially embedded into $\mathscr{A}$. It is also clear that $C$ (as well as $S$ ) is contained in the interior of $\mathscr{A}$.

Let us consider now the intersection of $\mathscr{A}$ with the first quadrant $Q_{1}$. The boundary of such intersection consists of two segments $L_{1}:=\left[r_{0}, 1\right] \times\{0\}, L_{2}:=\{0\} \times\left[r_{0}, 1\right]$ and two $\operatorname{arcs} C_{1}:=r_{0} S^{1} \cap Q_{1}, C_{2}:=S^{1} \cap Q_{1}$. We also define

$$
\mathscr{B}:=R^{-1}\left(\mathscr{A} \cap Q_{1}\right) \text {. }
$$

The set $\mathscr{B}$ is a topological rectangle for which we give an orientation by setting

$$
\begin{aligned}
& \mathscr{B}_{l}^{-}:=R^{-1}\left(L_{1}\right), \\
& \mathscr{B}_{r}^{-}:=R^{-1}\left(L_{2}\right), \\
& \mathscr{B}_{t}^{+}:=R^{-1}\left(C_{1}\right), \\
& \mathscr{B}_{b}^{+}:=R^{-1}\left(C_{2}\right) .
\end{aligned}
$$

By the assumptions, we see that

$$
\begin{gathered}
\|\Psi(z)\|>\|z\|, \quad \forall z \in \mathscr{B}_{l}^{-}, \\
\|\Psi(z)\|<\|z\|, \quad \forall z \in \mathscr{B}_{r}^{-} .
\end{gathered}
$$

Hence, on each path with values in $\mathscr{B}$ connecting $\mathscr{B}_{l}^{-}$with $\mathscr{B}_{r}^{-}$there is some point where $\|\Psi(z)\|=\|z\|$. Lemma 11 ensures the existence of a continuum $C^{\prime} \subset \mathscr{B}$ with $C^{\prime} \cap$ $\mathscr{B}_{b}^{+} \neq \emptyset, C^{\prime} \cap \mathscr{B}_{t}^{+} \neq \emptyset$, and $\|\Psi(z)\|=\|z\|$, for each $z \in C$.

We are now in a position to apply Lemma 18 which guarantees that $C^{\prime} \cap C \neq \emptyset$. By definition of $C$ and $C^{\prime}$ we conclude that any point $w \in C^{\prime} \cap C$ is a fixed point for $\Psi$ with $\Psi(w)=w \in \operatorname{int}\left(Q_{1} \cap \mathscr{A}\right)$. Repeating the same argument for the other quadrants, we find the remaining three fixed points.

We end the proof with a final comment about the "stability" of the existence result with respect to small perturbations. By this assertion, we mean that the existence of at least four fixed points is preserved if, instead $\Psi$, we take a continuous map which is sufficiently near to $\Psi$ in the $\|\cdot\|_{\infty}$-norm on $\mathscr{A}$. 
The reason for this fact comes from the strict inequalities that we have in (60) and (63). More precisely, the map $\Theta_{\psi}(z)-2 \pi$ is strictly positive on $\mathscr{A}_{i}$ and strictly negative on $\mathscr{A}_{0}$. Similarly, $\|\Psi(z)\|-\|z\|$, is strictly positive on one side of $\mathscr{B}$ and strictly negative on an opposite side of $\mathscr{B}$. Hence, the maps $\Theta_{\psi}(z)-$ $2 \pi$ and $\|\Psi(z)\|-\|z\|$ can be seen as the two components of a vector field, and such components change their sign passing from one side to the opposite side of a topological rectangle. In such a case, we can prove that the topological degree (the Brouwer degree) associated with such a vector field is nonzero (this is exactly what happens when we give a proof of the Poincaré-Miranda theorem using the Brouwer degree and assume that the vector field does not vanish on the boundary of the rectangle in order to have the topological degree well defined). In case of nonzero degree (which, one could prove, is our situation) it is known that the results about the existence of zeros (or, resp., fixed points) are stable with respect to small perturbations of the vector field. This is our case when the above strict inequalities hold.

Following the proof, it is clear that if $\theta_{0}>2 j \pi+\pi / 2$, for some positive integer $j$, then there are at least $4 j$ fixed points.

For a different application of these results to the existence of fixed points and periodic points to planar maps arising from ordinary differential equations, we refer also to [42].

\section{Acknowledgments}

The authors are sincerely grateful and indebted to the referee of their paper who checked very carefully their work and gave them extremely useful suggestions on how to correct some bugs in the original proofs and enhance the presentation.

\section{References}

[1] H. Poincaré, "Sur certaines solutions particulières du problème des trois corps," Comptes Rendus de l'Académie des Sciences, vol. 97, pp. 251-252, 1883.

[2] H. Poincaré, "Sur certaines solutions particulières du problème des trois corps," Bulletin Astronomique, vol. 1, pp. 65-74, 1884.

[3] F. E. Browder, "Fixed point theory and nonlinear problems," Bulletin of the American Mathematical Society, vol. 9, no. 1, pp. 1-39, 1983.

[4] W. Kulpa, "The Poincaré-Miranda theorem," The American Mathematical Monthly, vol. 104, no. 6, pp. 545-550, 1997.

[5] J. Mawhin, "Poincaré's early use of Analysis situs in nonlinear differential equations: variations around the theme of Kronecker's integral," Philosophia Scientiae, vol. 4, no. 1, pp. 103-143, 2000.

[6] H. W. Siegberg, "Some historical remarks concerning degree theory," The American Mathematical Monthly, vol. 88, no. 2, pp. 125-139, 1981.

[7] J. Mawhin, "Variations on Poincaré's-Miranda's Theorem," Advanced Nonlinear Studies, vol. 13, pp. 209-217, 2013.

[8] A. Pascoletti, M. Pireddu, and F. Zanolin, "Multiple periodic solutions and complex dynamics for second order ODEs via linked twist maps," in The 8th Colloquium on the Qualitative Theory of Differential Equations, vol. 8, Szeged, 2008, Electronic Journal of Qualitative Theory of Differential Equations, Szeged, vol. 14, pp. 1-32, 2008.
[9] W. Hurewicz and H. Wallman, Dimension Theory, vol. 4 of Princeton Mathematical Series, Princeton University Press, Princeton, NJ, USA, 1941.

[10] S. Alpern and A. Beck, "Hex games and twist maps on the annulus," The American Mathematical Monthly, vol. 98, no. 9, pp. 803-811, 1991.

[11] D. Gale, "The game of Hex and the Brouwer fixed-point theorem," The American Mathematical Monthly, vol. 86, no. 10, pp. 818-827, 1979.

[12] C. Conley, "An application of Ważewski's method to a nonlinear boundary value problem which arises in population genetics," Journal of Mathematical Biology, vol. 2, no. 3, pp. 241249,1975

[13] J. C. Alexander, "A primer on connectivity," in Fixed Point Theory, vol. 886 of Lecture Notes in Math, pp. 455-483, Springer, Berlin, Germany, 1981.

[14] G. J. Butler, "Rapid oscillation, nonextendability, and the existence of periodic solutions to second order nonlinear ordinary differential equations," Journal of Differential Equations, vol. 22, no. 2, pp. 467-477, 1976.

[15] C. Rebelo and F. Zanolin, "On the existence and multiplicity of branches of nodal solutions for a class of parameter-dependent Sturm-Liouville problems via the shooting map," Differential and Integral Equations, vol. 13, no. 10-12, pp. 1473-1502, 2000.

[16] A. Pascoletti and F. Zanolin, "A path crossing lemma and applications to nonlinear second order equations under slowly varying perturbations," Le Matematiche, vol. 65, no. 2, pp. 121$168,2010$.

[17] J. A. Kennedy and J. A. Yorke, “The topology of stirred fluids," Topology and Its Applications, vol. 80, no. 3, pp. 201-238, 1997.

[18] A. Berarducci, D. Dikranjan, and J. Pelant, "Uniform quasi components, thin spaces and compact separation," Topology and Its Applications, vol. 122, pp. 51-64, 2002.

[19] M. Dolcher, "Questioni di minimo per insiemi chiusi sconnettenti uno spazio topologico," The Mathematical Journal of the University of Padova, vol. 19, pp. 159-171, 1950.

[20] D. Papini and F. Zanolin, "Fixed points, periodic points, and coin-tossing sequences for mappings defined on twodimensional cells," Fixed Point Theory and Applications, vol. 2004, no. 2, pp. 113-134, 2004.

[21] A. Pascoletti and F. Zanolin, "Example of a suspension bridge ODE model exhibiting chaotic dynamics: a topological approach," Journal of Mathematical Analysis and Applications, vol. 339, no. 2, pp. 1179-1198, 2008.

[22] M. Pireddu and F. Zanolin, "Cutting surfaces and applications to periodic points and chaotic-like dynamics," Topological Methods in Nonlinear Analysis, vol. 30, no. 2, pp. 279-319, 2007.

[23] E. E. Moise, Geometric Topology in Dimensions 2 and 3, vol. 47 of Graduate Texts in Mathematics, Springer, New York, NY, USA, 1977.

[24] J. S. Muldowney and D. Willett, "An elementary proof of the existence of solutions to second order nonlinear boundary value problems," SIAM Journal on Mathematical Analysis, vol. 5, pp. 701-707, 1974.

[25] B. R. Gelbaum and J. M. H. Olmsted, Counterexamples in Analysis, Dover, Mineola, NY, USA, 2003.

[26] A. Pascoletti and F. Zanolin, "Chaotic dynamics in periodically forced asymmetric ordinary differential equations," Journal of Mathematical Analysis and Applications, vol. 352, no. 2, pp. 890906, 2009. 
[27] J. W. Alexander, "A proof of Jordan's theorem about a simple closed curve," Annals of Mathematics, vol. 21, no. 3, pp. 180-184, 1920.

[28] J. W. Alexander, "A proof and extension of the Jordan-Brouwer separation theorem," Transactions of the American Mathematical Society, vol. 23, no. 4, pp. 333-349, 1922.

[29] M. H. A. Newman, Elements of the Topology of Plane Sets of Points, Cambridge University Press, New York, NY, USA, 1961.

[30] D. E. Sanderson, "Advanced plane topology from an elementary standpoint," Mathematics Magazine, vol. 53, no. 2, pp. 81-89, 1980.

[31] P. A. Smith, "Book review: elements of the topology of plane sets of points," Bulletin of the American Mathematical Society, vol. 45, no. 11, pp. 822-824, 1939.

[32] S. P. Hastings, "An existence theorem for a problem from boundary layer theory," Archive for Rational Mechanics and Analysis, vol. 33, pp. 103-109, 1969.

[33] S. Hastings, “The existence of periodic solutions to Nagumo's equation," The Quarterly Journal of Mathematics, vol. 25, pp. 369-378, 1974.

[34] S. P. Hastings, "On the existence of homoclinic and periodic orbits for the Fitzhugh-Nagumo equations," The Quarterly Journal of Mathematics, vol. 27, no. 105, pp. 123-134, 1976.

[35] J. B. McLeod and J. Serrin, "The existence of similiar solutions for some laminar boundary layer problems," Archive for Rational Mechanics and Analysis, vol. 31, pp. 288-303, 1969.

[36] R. E. L. Turner, "Nonlinear eigenvalue problems with applications to elliptic equations," Archive for Rational Mechanics and Analysis, vol. 42, pp. 184-193, 1971.

[37] P. Le Calvez, M. Martens, C. Tresser, and P. A. Worfolk, "Stably nonsynchronizable maps of the plane," Nonlinearity, vol. 12, no. 1, pp. 9-18, 1999.

[38] M. Kallipoliti and P. Papasoglu, "Simply connected homogeneous continua are not separated by arcs," Topology and Its Applications, vol. 154, no. 17, pp. 3039-3047, 2007.

[39] M. Henle, A Combinatorial Introduction to Topology, Dover, New York, NY, USA, 1994.

[40] K. Kuratowski, Topology. Vol. I, Academic Press, New York, NY, USA, 1966.

[41] J. G. Hocking and G. S. Young, Topology, Dover, New York, NY, USA, 2nd edition, 1988.

[42] A. Pascoletti and F. Zanolin, "A topological approach to bendtwist maps with applications," International Journal of Differential Equations, vol. 2011, Article ID 612041, 20 pages, 2011.

[43] J. P. LaSalle, The Stability of Dynamical Systems, SIAM, Philadelphia, Pa, USA, 1976. 


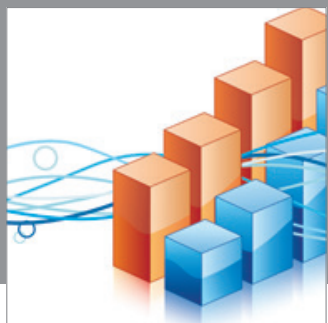

Advances in

Operations Research

mansans

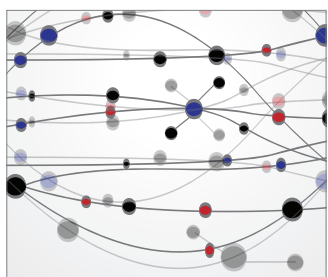

The Scientific World Journal
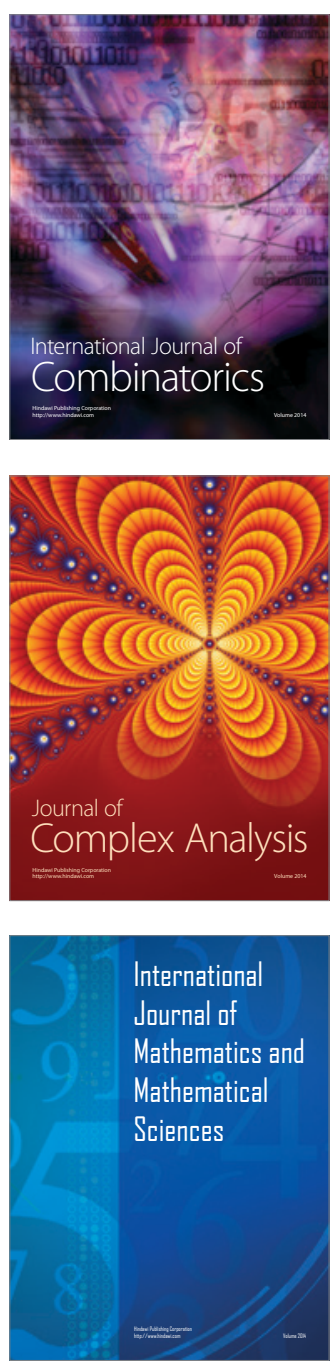
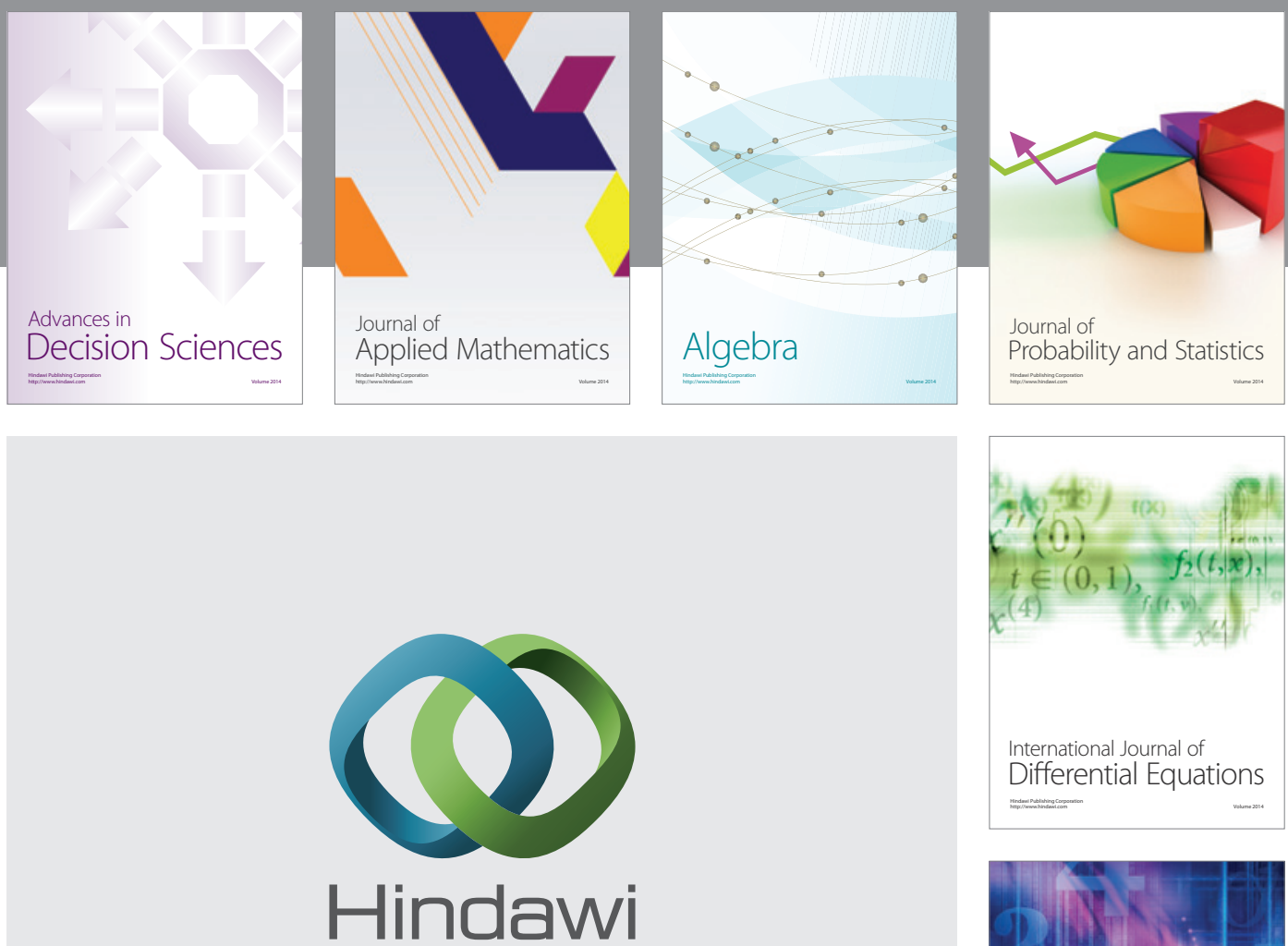

Submit your manuscripts at http://www.hindawi.com
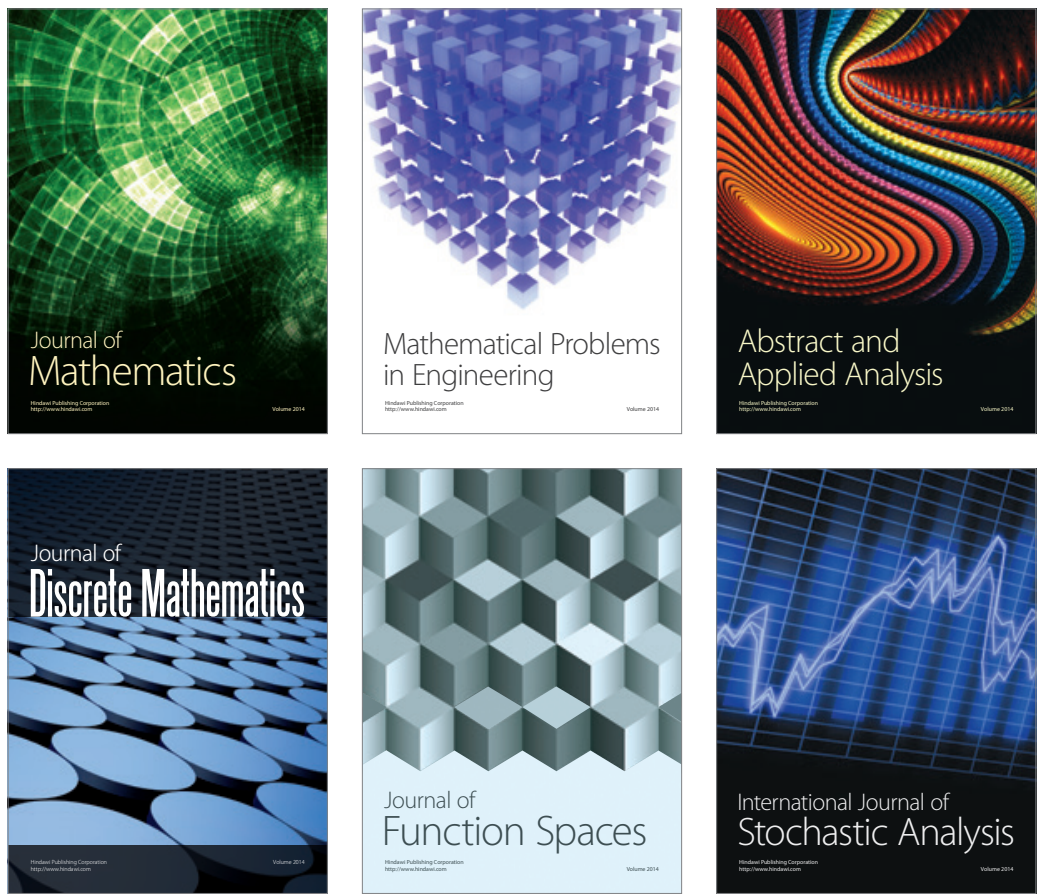

Journal of

Function Spaces

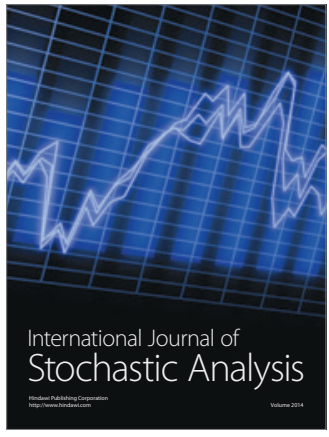

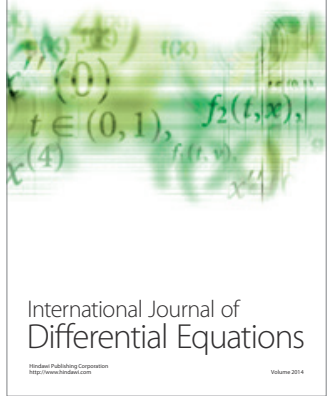
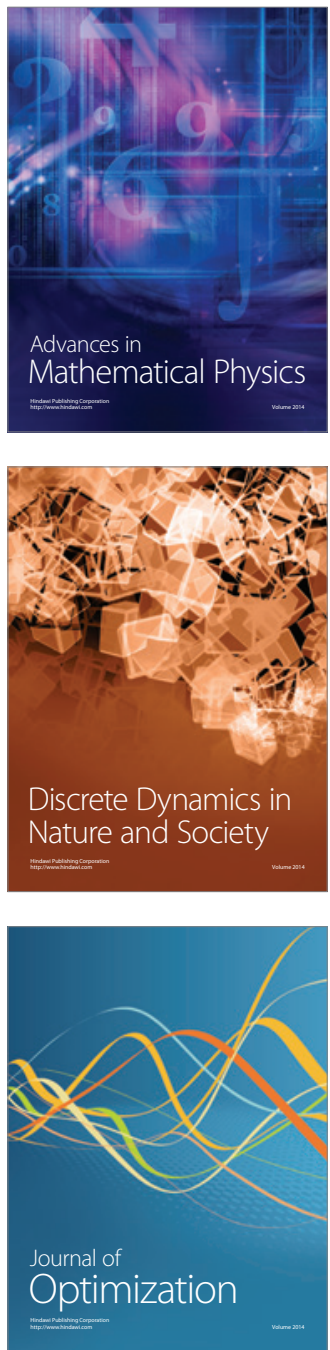